\title{
Análise Comportamental de Problemas de Interação Social de Indivíduos com Psoríase
}

\author{
Carolina Martinez Sampaio Mota \\ Márcia Cristina Caserta Gon \\ Airton dos Santos Gon \\ Universidade Estadual de Londrina
}

\begin{abstract}
RESUMO
Psoríase é uma doença crônica que acomete a pele de indivíduos de ambos os sexos a partir da segunda década de vida. Além do desconforto físico decorrente de sintomas como descamação e prurido, queixas referentes a dificuldades interpessoais são relatadas com frequência por pacientes que buscam atendimento médico. $\mathrm{O}$ indivíduo com psoríase pode apresentar dificuldades de interação social em diversos contextos pela reação negativa dos outros à aparência inestética das lesões. Por esta razão, este indivíduo tenta fugir ou esquivar-se de situações em que exista a possibilidade de exposição física, com o intuito de escapar aos olhares constantes e comentários das outras pessoas em relação à sua aparência. Quando isto não é possível, procura ocultar as lesões por meio do uso de cosméticos (camuflagem) ou de roupas mais fechadas, mesmo em dias de clima quente. Neste artigo, os autores avaliam como a análise do comportamento entende comportamento social e discutem, segundo esse enfoque psicológico, como possíveis variáveis ambientais podem controlá-lo, alterando a probabilidade de sua ocorrência em diferentes contextos sociais.
\end{abstract}

Palavras-chave: psoríase; interação social; análise do comportamento.

\begin{abstract}
Behavioral Analysis of Social Interaction Problems of Individuals With Psoriasis

Psoriasis is a chronic skin disease that affects individuals of both genders beginning from the second decade of life. Besides physical discomfort due the symptoms like itching and scaling, interpersonal difficulties are commonly reported by patients to their doctors. The unaesthetic aspect of psoriatic lesions usually affects the patients' ability to deal with negative reactions from other people, in several contexts of social interactions. Therefore, individuals try to escape from situations where they are at risk of being physically exposed, in an attempt to avoid being stared at and comments on their appearance. They also tend to cover lesions, using cosmetic camouflage or protective clothes like long sleeves, skirts and caps, even on hot days. In this article, the authors evaluate how behavior analysis explains social behavior and they discuss, using this approach, how environmental variables can control social behavior, changing the probability of its occurrence in different social contexts.
\end{abstract}

Keywords: psoriasis; social interactions; behavior analysis.

A maioria das pesquisas sobre psoríase mostra que, além do desconforto físico decorrente dos sintomas como prurido, descamações, lesões na pele e dos tratamentos que nem sempre produzem resultados esperados, os indivíduos com esta doença ainda sofrem com as reações de preconceito de outras pessoas devido à aparência inestética de sua pele, tais como olhares, comentários insistentes sobre a doença, afastamento, críticas à sua aparência, entre outras. Assim, um indivíduo que possui a superfície da pele visivel- mente alterada pela psoríase poderá ser afetado diretamente não apenas pela sua condição biológica propriamente dita, mas também pela maneira como outras pessoas reagem à sua aparência física em diversos contextos sociais, como família, escola, trabalho e outros.

Por esta razão, queixas referentes às dificuldades interpessoais são relatadas frequentemente pelos pacientes com psoríase aos seus médicos. Porém, apesar de reconhecerem a importância da relação entre pro- 
blemas psicológicos e afecções cutâneas, discuti-la e ajudar essas pessoas a compreendê-la é uma tarefa difícil para muitos destes profissionais (Gupta \& Gupta, 2004; Mingnorance, Loureiro \& Okino, 2002; Picardi \& Abeni, 2001a; Picardi \& Abeni, 2001b). Apesar da relevância do tema "dificuldades interpessoais e doenças crônicas de pele", há poucas pesquisas nacionais que o discutem. Sobretudo, há escassez de trabalhos cuja referida temática seja debatida por analistas do comportamento.

Diante desta perspectiva, no presente artigo, os autores têm os objetivos de: (1) discutir a importância da pele nas relações interpessoais e nos processos de socialização para aqueles indivíduos com alterações dermatológicas; (2) apresentar aspectos médicos da psoríase e de seu tratamento; (3) apresentar alguns estudos correlacionados sobre psoríase e dificuldades de interação social e (4) discutir como a análise do comportamento considera as dificuldades de interação social apresentadas por estes indivíduos, as quais são sugeridas em pesquisas da área.

\section{Importância da pele nas relações interpessoais e nos processos de socialização de indivíduos com alterações dermatológicas}

A pele é um dos principais órgãos sensoriais dos seres humanos. Ela constitui o órgão dos sentidos mais extenso e fundamental, pois envolve o corpo por completo e é o primeiro sistema sensorial a se desenvolver no embrião humano e a se tornar funcional (Montagu, 1986). A pele está intimamente relacionada com a sensibilidade corporal, pois sob ela estão localizados receptores térmicos, táteis e de pressão que são os mecanismos de percepção e contato com o mundo externo ao corpo. Os olhos, o ouvido, o nariz e a boca são formados também a partir de modificações do tecido epidérmico (Knobe, 2004). Ainda, a pele tem a mesma origem embrionária do sistema nervoso central (o ectoderma), possuindo por isso, receptores nervosos e neurotransmissores em comum com este sistema (Picardi \& Abeni, 2001a). Muitos profissionais da saúde consideram a pele como o "espelho" do funcionamento do organismo, na medida em que as características epidérmicas como cor, textura, elasticidade, umidade e secura fornecem informações sobre o estado comportamental e fisiológico do indivíduo (Knobe, 2004; Montagu, 1986).

Além da importância fundamental de sua fisiologia, a pele também apresenta uma importante função na socialização dos seres humanos, pois se trata do revestimento corporal de maior exposição e visibilidade para o indivíduo e para o grupo social no qual ele está inserido (Montagu, 1986). O revestimento cutâneo do corpo define a aparência física e participa prioritariamente na construção da identidade pessoal dos indivíduos. As pessoas se reconhecem pela aparência física, assim como são reconhecidas pela apresentação pessoal conferida pelas características de sua pele.

Portanto, são os grupos sociais que constroem significados estéticos quanto à aparência física da pele. Desse modo, muitas pessoas que apresentam características cutâneas as quais não se enquadram nos padrões estéticos definidos por um determinado grupo social podem ser expostas a reações de preconceito e de estigmatização (Silva \& Silva, 2007). Críticas, brincadeiras e apelidos ofensivos podem trazer grande incômodo para as pessoas com deformidades, manchas e lesões na pele. Geralmente estas se queixam de sua aparência, consideram-se feias e relatam sentir constrangimento nas relações interpessoais, por isso, frequentemente evitam contatos sociais, o que afeta profundamente sua vida, com prejuízos pessoais, profissionais e afetivos (Mingnorance \& cols., 2002). Em face destas queixas, muitos indivíduos que têm a configuração da pele alterada por afecções dermatológicas, além de sofrerem com o desconforto físico ocasionado pelas lesões, também vivenciam muitas dificuldades de interações sociais, o que também ocorre no caso da psoríase.

\section{Psoríase: características e tratamento}

A psoríase é uma doença crônica comum, caracterizada por lesões de pele eritemato-escamosas (Koo, Do \& Lee, 2000; Krueger e cols., 2001; Seng \& Nee, 1997). As lesões podem ocorrer em qualquer parte do corpo, mas aparecem com maior frequência em determinadas áreas como couro cabeludo, cotovelos e joelhos. Pode haver comprometimento das unhas, palmas e plantas (Picardi \& Abeni, 2001a). Pacientes com psoríase frequentemente apresentam sintomas como prurido, ardência e sensibilidade dolorosa das lesões, bem como dores articulares e artrite (Picardi \& cols., 2005; Takahashi, 1991).

A causa é desconhecida, podendo atingir ambos os sexos, em qualquer idade, sendo mais frequente nas segunda e terceira décadas de vida (Finlay, 2001; Savin \& Cotteril, 1992; Zacharie, Oster, Bjerring \& Kragballe, 1996). É uma doença de herança multifatorial, cuja expressão está relacionada a fatores de natureza poligênica, ambiental ${ }^{1}$ e psicológica ${ }^{2}$ (Abadie, 
Kent \& Gawkrodger, 1994; Castro, Andrade \& Muller, 2006; Gupta \& Gupta, 2004; Hoffman, Zogbi, Fleck \& Muller, 2006; Koo \& col., 2000; Ongenae, Beelaert, Geel \& Naeyaert, 2006). Embora a causa da psoríase seja desconhecida, as lesões são caracterizadas por crescimento rápido da epiderme associado com aumento na velocidade de troca das células da epiderme. Em condições normais, as células normais da pele são substituídas em ciclos de cerca de 28 dias. $\mathrm{Na}$ psoríase, células epidérmicas são substituídas em apenas quatro dias. Alguns fatores podem atuar como agravantes na psoríase, incluindo infecções, clima frio e alguns medicamentos como betabloqueadores e lítio. Além destes, estresse, obesidade e alcoolismo têm também sido implicados na exacerbação do quadro de psoríase.

Para o tratamento da psoríase é importante determinar a extensão de acometimento da doença. Quando ela é localizada, o tratamento pode ser conduzido com uso de medicamentos tópicos (cremes e pomadas) (Koblenzer, 1995; Koblenzer, 1996; Marot, 1993; Taylor, 1997), mas se está generalizada pelo corpo é necessária a utilização de métodos de tratamento sistêmico (Bilks \& Mark, 1990; Capoore, Payne \& Goldin, 1998; Patrus, 1991). Exemplos de tratamentos na psoríase generalizada incluem a fototerapia com uso de luzes ultravioleta, e drogas anti-metabólicas e imunossupressores. Mas nem sempre o tratamento apresenta o resultado esperado pelos pacientes, que geralmente abandonam as prescrições médicas pela demora no controle parcial ou total dos sintomas ou pelo alto custo financeiro dos medicamentos (Fried, Gupta \& Gupta, 2005; Renzi \& cols., 2001)

Os sintomas da psoríase, como descamações e prurido, não apenas produzem desconforto físico, mas também podem levar o indivíduo a apresentar muitas dificuldades em situações sociais nas quais há exposição total ou parcial de seu corpo (Ginsburg \& Link, 1988; Picardi \& Abeni, 2001b). Em geral, indivíduos com psoríase tendem a evitar mais as situações sociais de exposição do corpo quanto maior for a quantidade e/ou a extensão das lesões; quando a região do corpo atingida é mais visível (cabeça, pescoço e mãos); quando a duração da doença é prolongada e quanto mais jovem eles forem. (Benrud-Larson \& cols., 2003; Mingnorance \& cols., 2002).

\section{Psoríase e interações sociais de estigmatização}

Muitos estudos correlacionados sugerem que indivíduos com psoríase se queixam de se sentir estigmatizados (Finlay, 2001; Fried \& cols., 2005; Ginsburg
\& Link, 1988; Ongenae \& cols., 2006). Por exemplo, Ginsburg e Link (1988) procuraram operacionalizar o conceito de estigmatização por meio de um estudo com 100 adultos com psoríase. Eles identificaram seis dimensões da experiência de estigma que foram: sentimentos de antecipação da rejeição; de ser defeituoso; sensibilidade ao comportamento/reação de outros perante a sua doença (lesões na pele); culpa; vergonha e práticas de uso de camuflagem, como cobrir o corpo ou passar maquiagem. Os resultados mostraram marcada variabilidade na presença e na magnitude das respostas de estigmatização e de isolamento de acordo com a idade dos participantes, da extensão da lesão, do status de seu emprego, da duração da doença e de situações de rejeição a que o participante foi exposto (abandono de pessoas próximas, ser excluído de um grupo, ser criticado verbalmente, ter os seus convites recusados por pessoas ou permanecer isolado). De todos os aspectos da doença, a quantidade, a visibilidade e a extensão das lesões no corpo foram os fatores mais relacionados à resposta de estigmatização e de isolamento.

Apesar de a psoríase ser uma inflamação epidérmica puramente cutânea e não contagiosa, a maioria dos indivíduos que a apresentam se queixam de inibição quando precisam expor seu corpo, relatam se sentir insatisfeitos, descontentes e revoltados com sua aparência física. Eles também se sentem rejeitados, defeituosos, envergonhados, culpados e estigmatizados quando interagem socialmente com pessoas que não apresentam doença de pele (Finlay, 2001; Fortune, Richards, Main \& Grifiths, 1998; Fried \& cols., 2005). Muitos destes indivíduos relacionam sua insatisfação às demonstrações verbais ou às expressões faciais de repulsa; afastamento de pessoas desconhecidas, olhares e comentários insistentes sobre a doença, apelidos ou piadas sobre sua pele (Leary, Rapp, Herbest, Exum \& Feldman, 1998; Vardy \& cols., 2002). Outros relatam, ainda, que a vergonha e a revolta que sentem em relação a sua aparência física estão relacionadas à rejeição dos parceiros nos relacionamentos afetivos mais íntimos (Benrud-Larson \& cols., 2003; Finlay, 2001).

Alguns comportamentos de preconceito relatados também nestas pesquisas são sutis ou menos evidentes, tais como evitar o toque e a proximidade física com indivíduos que apresentam a doença, como, por exemplo, sentar junto, abraçar, pegar na mão, usar o mesmo sabonete ou toalha, compartilhar o uso de objetos. Há, ainda, formas mais indiretas de demonstração de preconceito, como ocorre quando uma pes- 
soa com psoríase fica sabendo que seus colegas de classe fazem piadas de sua pele quando não está presente (Leary \& cols., 1998; Mingnorance \& cols., 2002; Vardy \& cols., 2002). Deste modo, a exposição a situações de interação social que envolvem preconceito, tais como as mencionadas no parágrafo anterior, pode contribuir para que os indivíduos com psoríase as evitem com mais frequência.

Tem sido mostrado também nas pesquisas sobre estigmatização de indivíduos com psoríase que é na faixa etária entre 18 e 45 que são relatadas maiores dificuldades de relacionamento interpessoal (Mingnorance \& cols., 2002; Rapp, Feldman, Exum, Fleischer \& Reboussin, 1999; Whal, Loge, Wiklund; Hanestad $\&$ Trondheim, 2000). Isto ocorreria porque é neste período da vida em que a exposição a situações novas e a pessoas desconhecidas é mais frequente e tem maior relevância, devido às exigências profissionais $\mathrm{e}$ sociais. Assim, indivíduos com esta doença estão expostos constantemente a situações que exigem que eles iniciem e mantenham conversas face a face, conversem com o sexo oposto e com pessoas desconhecidas, realizem convites, dialoguem, enfrentem críticas aos seus trabalhos e defendam suas opiniões, mesmo que sejam contrárias às opiniões de pessoas que representam autoridades (Amaral, Bravo \& Messias, 1996).

Embora a correlação entre dificuldade de exposição do corpo e psoríase possa ser sugerida nos estudos citados acima, parece não haver clareza se tal exposição se tornou aversiva a partir do aparecimento da doença da pele ou se o individuo já apresentava dificuldades em se expor fisicamente antes da sua manifestação (Benrud-Larson \& cols., 2003; Rapp, Cottrell \& Leary, 2001). Mesmo assim, o que é importante destacar nestas pesquisas é que, apesar de não constarem informações sobre o comportamento do indivíduo em relação à exposição de seu corpo a outras pessoas antes do aparecimento dos sintomas da doença, aqueles cujas lesões são maiores procuram se afastar do convívio com outros, evitando principalmente situações nas quais tenham que se expor fisicamente (esquiva de exposição pública) (Leary \& cols., 1998; Vardy \& cols., 2002). Em virtude destas dificuldades, eles procuram evitar com mais frequência atividades rotineiras e de lazer (Fortune \& cols., 1998; Patrus, 1991; Rosmaninho \& cols., 2003). Muitos outros também costumam evitar contatos mais íntimos com o sexo oposto e apresentam muitos problemas sexuais, tais como, falta de interesse sexual, anorgasmia, frigidez, entre outras, sendo destacada a necessidade de suporte psicológico para estes casos (Porter, Beuf,
Lerner \& Nordlund, 1990; Taborda, Weber \& Freitas, 2005; Vardy \& cols., 2002).

Se as interações sociais e a exposição pública do corpo parecem ser condições aversivas das quais os indivíduos com psoríase tendem a se esquivar, é importante avaliar como a análise do comportamento entende comportamento social e discutir, segundo esse enfoque psicológico, como possíveis variáveis ambientais podem controlá-lo, alterando a probabilidade de sua ocorrência em diferentes contextos sociais.

\section{Interações sociais na análise do comportamento}

Conforme Skinner, (1953/1965) um comportamento deve ser considerado social quando envolve duas ou mais pessoas e quando o comportamento de uma delas, para produzir o efeito sobre o ambiente, depende do comportamento da outra. Assim, a interação entre as pessoas que se comportam socialmente compõe um ambiente social e este ambiente seleciona os comportamentos dos indivíduos que nele se comportam (Hayes, 1987; Skinner, 1953/1965). Deste modo, o comportamento social ocorre porque um organismo é importante para outro, como parte integrante de seu próprio ambiente (Skinner, 1953/1965). Neste sentido, o reforço em um ambiente é considerado social porque é mediado por outra pessoa. Assim, se não houver a mediação do outro indivíduo, os comportamentos que dependem de reforço social não apresentam nenhum efeito sobre o ambiente.

Embora as contingências de reforço possam modelar comportamentos sociais bastante complexos, para que isso ocorra é preciso que haja a emissão de alguns comportamentos que serão aprimorados. Inicialmente, os critérios para que o comportamento seja reforçado positivamente são mais simples, e, gradualmente aumentam os requisitos para que ele possa ser reforçado novamente (Skinner, 1953/1965). Ou seja, os comportamentos tornam-se complexos porque as contingências de reforço partem de comportamentos menos rebuscados, que são modelados por aproximações sucessivas e reforço diferencial. Sem essa história de modelagem, muitas contingências sociais de reforço seriam ineficazes em produzir comportamento social (Hayes, 1987; Skinner, 1953/1965). Por exemplo, a criança aprende a falar primeiramente balbuciando sons que se assemelham à fala adulta e os pais, prontamente respondem a esse balbucio com palavras ou entregando objetos ao bebê. Porém, quando as crianças estão maiores, com quatro ou cinco anos, aquele balbucio não mais se faz efetivo, os pais corrigem os filhos quando estes falam errado e pedem para que 
eles soletrem as palavras. Neste sentido, ao ensinar indivíduos que apresentam dificuldades em interações sociais, parte-se do conjunto de comportamentos que eles já apresentam, e reforçam-se variações destes comportamentos.

Porém, a dependência da mediação de outras pessoas pode implicar em alguns problemas produzidos pelo reforço social, os quais não são observados em ambientes não sociais. Estes problemas ocorreriam porque nas contingências sociais a liberação do reforço nem sempre depende apenas da apresentação do comportamento a ser reforçado, mas também do agente que libera o reforço. Por causa desta dependência, o reforço social varia de momento a momento, o que implica que, conforme a ocasião, uma dada resposta pode ser seguida por diferentes consequências ou que diferentes respostas podem produzir a mesma consequência (Skinner, 1953/1965). Por exemplo, considere um adolescente com psoríase cujo pai, quando o jovem não apresenta lesões, oferece atenção ao ser obediente e aplicado aos estudos, mas quando tem as lesões no corpo, lhe oferece atenção, independentemente do comportamento. Se a atenção funcionar como reforço positivo para o adolescente, não ocorrerá a diferenciação entre ambos os comportamentos (o socialmente aceitável - estudar e obedecer e o socialmente não aceitável - não estudar e desobedecer ao pai). Além disso, quando o adolescente apresenta as lesões, a liberação do reforço não depende do seu comportamento, mas de contingências que envolvem exclusivamente o pai, que diante das lesões, pode sentir pena e lhe oferece atenção.

Dessa forma, o ajustamento social relaciona-se diretamente com a funcionalidade do comportamento e não apenas com a sua topografia. Como afirma Skinner: "Um estímulo social, como qualquer outro estímulo, torna-se importante ao controlar o comportamento por causa das contingências nas quais ele está inserido" (Skinner, 1953/1965, p. 299). Estas consequências podem ser imediatas ou ocorrer a longo prazo. O critério para avaliar a eficácia de um comportamento nas interações sociais são as consequências produzidas por esse comportamento não só com relação a como elas retroagem sobre o comportamento do próprio indivíduo que se comporta, mas também com relação aos seus efeitos sobre os demais indivíduos da interação social. Tomando-se por base a interação descrita no parágrafo anterior entre o adolescente com psoríase e seu pai, se o filho passar a responder discriminativamente às contingências que mantém o comportamento de atenção e afeto do pai, ele poderá tentar controlá-los através de sua doença, queixando-se mais dos sintomas diante do pai ou até mesmo não seguindo corretamente o tratamento prescrito, por exemplo. Por outro lado, este processo de discriminação nem sempre é facilmente aprendido. Primeiro, porque os estímulos sociais não apresentam propriedades físicas facilmente observadas. Segundo, porque eles dependem das práticas culturais e da história pessoal de exposição a estes estímulos (Skinner, 1953/1965).

Para se analisar melhor esta dificuldade tem-se a expressão facial de sorriso como exemplo. Em determinadas circunstâncias, sua apresentação funciona como ocasião na qual certos tipos de comportamentos sociais são mais prováveis de ser reforçados. Exemplificando, quando uma pessoa desconhecida sorri para outra é mais provável que ela permita proximidade física e será mais receptiva para conversar, caso a outra se aproxime dela. Mesmo existindo uma variabilidade muito grande de topografias do sorrir, elas constituem uma mesma classe se produzirem as mesmas consequências, isto é, aproximação física do outro e início de uma conversa (Baum, 1994/1999).

Porém, nem sempre um indivíduo terá seu comportamento reforçado positivamente ao se expor à situação social e identificar determinados estímulos sociais. Novamente, tem-se o sorriso como exemplo. Em determinadas circunstâncias, ele pode não ser seguido de reforço positivo ou poderá ser punido. $\mathrm{O}$ sorriso de uma pessoa que sinalizou alta probabilidade de aproximação e de conversa pode, quando apresentado por outra ou pela mesma pessoa em contexto diferente, ser considerado malicioso e, a pessoa que sorriu, provavelmente será criticada verbalmente. Neste contexto, o sorriso será punido. É importante enfatizar que um dado evento não apresenta a função de estímulo social em si mesmo. Ele passou a exercer esta função por causa da interação entre um organismo e outro e sob determinadas circunstâncias (Abib, 2004; Skinner, 1953/1965). Os indivíduos passam a responder a determinados eventos como estímulos sociais, que sinalizam determinadas consequências, em razão de uma história prévia de interação com estes eventos. Nesta história, o evento deixa de ser neutro e se torna um estímulo discriminativo para a ocorrência de determinados comportamentos.

Concluindo, estímulos sociais não sinalizam consequências certas e evidentes porque as contingências sociais são dinâmicas e muito variáveis, dependendo dos contextos. Além disso, elas não são compostas apenas por reforçadores sociais positivos, como aproximação, atenção, aprovação e afeto, mas também por 
reforçadores sociais negativos, tais como afastamento, reprovação, ridicularização e insulto. Se a possibilidade de ridicularização ou de insulto é aumentada quando os indivíduos apresentam psoríase, a exposição social para eles tornar-se-á ainda mais provável de ser punida devido à configuração inestética da pele. Nesta condição, comportamentos de fuga e de esquiva têm maior probabilidade de ocorrer quando comparados aos de pessoas que não têm uma doença de pele.

\section{Padrões de fuga-esquiva de interações sociais e suas consequências para os indivíduos com psoríase}

Como destacado nas seções anteriores, é mais comum os indivíduos com psoríase queixarem-se da avaliação e do julgamento de outras pessoas, sentindo-se mais estigmatizados do que pessoas que não apresentem qualquer tipo de desfiguração da pele (Ginsburg \& Link, 1988; Ongenae \& cols., 2006). Sendo assim, o julgamento e a avaliação negativa de outras pessoas poderiam constituir situações aversivas que os indivíduos com esta doença possivelmente já vivenciaram diretamente em contextos sociais diferentes. Se isto aconteceu, será mais provável que venham a evitar o contato com situações semelhantes novamente. Se não for possível evitá-las, eles poderão não apresentar os comportamentos que foram punidos na situação passada, como, por exemplo, iniciar uma conversa com uma pessoa estranha porque quando o fez ouviu um comentário negativo sobre sua doença. Assim, os efeitos punitivos produzidos pela situação aversiva podem suprimir apenas comportamento punido, mas não estabelecem contingências nas quais o indivíduo possa se comportar de modo mais eficiente, ou seja, o que ele aprende é como não se comportar (Sidman, 1989/1995; Skinner, 1953/1965).

Mas, além de ocorrer a supressão do comportamento punido anteriormente, estímulos aversivos produzem reações emocionais perturbadoras e indesejáveis. Porém, neste caso, o próprio corpo produz a estimulação aversiva e, como não há fuga ou esquiva apropriada desta estimulação corporal, a punição se torna uma condição crônica. Quando a punição se dirige aos comportamentos emocionais reflexos, não é possível apresentar respondentes incompatíveis com os punidos e como afirma Skinner (1953/1965): "como não se trata de um operante, não há como executar exatamente o contrário" (p. 191). Assim, a exposição direta à situação aversiva sem possibilidade de fuga poderá levar o indivíduo a apresentar reações respondentes fortíssimas, as quais interferirão na ati- vidade operante em curso (supressão condicionada) e levar à redução no responder operante (Millenson, 1967/1975; Zamignani \& Banaco, 2004). Por exemplo, eles param de fazer o que estavam fazendo, ficando sob o controle de reflexos, como taquicardia, enjôo, dor no peito, frio na barriga, nó na garganta, mãos suadas e tremores (Zamignani \& Banaco, 2004).

Ocorre também, se a estimulação aversiva for muito intensa, qualquer sinal do ambiente que indique a possibilidade de punição poderá controlar o comportamento, levando o indivíduo se manter em "estado de alerta" constante (Sidman, 1989/1995). Nestas condições, os subprodutos emocionais experimentados por estes indivíduos são estados de ansiedade generalizada e sentimentos de impotência (Catania, 1999; Michael, 1975; Perone, 2003; Sidman, 1989/ 1995). Mas, se além de intensa, a apresentação de estímulos aversivos for frequente e inesperada e o responder não tiver como consequência a cancelamento ou atraso desta estimulação, um estado de desamparo é produzido. Se isto acontecer o indivíduo pode ficar "paralisado" ou "passivo", deixando de se comportar na situação (Hünziker, 1997; Michael, 1975). Esta "inatividade" torna-se incompatível com a possibilidade de aprender comportamentos alternativos mais eficazes naquela situação (Skinner, 1953/ 1965).

Quando, porém, a fuga destas situações é possível, o indivíduo fará o possível para evitá-las futuramente. Ao agir assim, ele também não aprende como se comportar nelas (Hayes, 1987). A esquiva se tornaria generalizada e poderá assumir dimensões cada vez maiores. Ao evitar alguns contextos com muita frequência o indivíduo terá menos oportunidades para produzir reforçadores positivos, empobrecendo seus repertórios de relacionamentos interpessoais e profissionais, entre outros (Cordova \& Scott, 2001).

Por estas razões, ao evitarem interagir com outras pessoas e, consequentemente fugir ou esquivar-se de contingências potencialmente aversivas que podem ocorrer nestas interações, indivíduos com psoríase poderão generalizar estes comportamentos para muitas situações sociais. Esta generalização pode produzir passividade ou "déficit comportamental", já que, ao evitar situações aversivas, perde-se a oportunidade de adquirir novos comportamentos pró-sociais e, consequentemente perde-se o acesso a reforçadores sociais positivos importantes como atenção, afeto e aprovação (Sidman, 1989/1995; Skinner, 1953/1965). Em virtude disso, pessoas que não foram expostas a determinados estímulos sociais ou evitam se expor a 
contextos sociais nos quais tais estímulos estão presentes, não têm a oportunidade de apresentar comportamentos que possam ser reforçados positivamente nestes contextos (Hayes, 1987). Esta perda de reforçadores, assim como a perda de oportunidades para se comportar eficazmente podem explicar as dificuldades e prejuízos nos relacionamentos sociais, profissionais e afetivos de indivíduos com psoríase que são relatadas nas diversas pesquisas realizadas sobre o tema. Mas, enquanto a fuga ou esquiva destes contextos diminui a variabilidade comportamental, a exposição a eles contribui substancialmente para a modelagem dos repertórios de enfrentamento às estimulações aversivas decorrentes da doença de pele como os incômodos físicos ou as críticas. Nesta perspectiva é provável que os indivíduos com psoríase em isolamento social sejam mais vulneráveis à depressão e ansiedade, porque dispõem de menos contextos reforçadores e de menos possibilidade de enfrentar as situações aversivas.

Por outro lado, os comportamentos de fuga e de esquiva podem ficar não apenas sob o controle da retirada ou da interrupção da estimulação aversiva, mas também de consequências reforçadoras positivas que seguem a sua emissão (Zamignani \& Banaco, 2004). Por exemplo, quando pessoas com psoríase evitam contato com desconhecidos temendo reações negativas em relação a sua aparência e recebem o carinho e o consolo dos familiares por isso. Ou quando, ao evitarem situações de contato interpessoal, acabam também se esquivando de outras situações igualmente aversivas, como quando um empregado pede ao seu chefe para não participar de reuniões que considera enfadonhas e alega vergonha de sua pele para não comparecer. Se o chefe concorda, o empregado não só evita se expor aos olhares de pessoas menos conhecidas (situação aversiva), assim como evita a reunião. Em ambos os exemplos, o reforço positivo também não favorece a aprendizagem de comportamentos alternativos mais eficazes para enfrentar estas situações.

Como discutido anteriormente, padrões comportamentais de fuga ou esquiva das situações sociais parecem comprometer não só a exposição pública, a vida social e a convivência em grupo em uma perspectiva mais ampla, mas também a convivência entre parceiros, na vida íntima, nos relacionamentos amorosos e nos familiares. Tais comportamentos ocorreriam com mais frequência nas situações que exigem maior exposição do indivíduo, seja a exposição de seus sentimentos, pensamentos ou a de seu corpo (Cordova \& Scott, 2001). Isso pode ocorrer porque o ingresso em interações sociais e íntimas parece exigir a apresentação de comportamentos com alta probabilidade de serem punidos, tais como: demonstrar sentimentos (tristeza, mágoa, medo, amor, vergonha, atração); ou afeto (abraçar e beijar as pessoas); contar segredos; envolver-se em atividade sexual e despir-se. Para emitir esses comportamentos, é preciso que os indivíduos tenham desenvolvido tolerância à rejeição ou à avaliação negativa (Cordova \& Scott, 2001). Quando em vez de punidos, tais comportamentos são reforçados positivamente, estabelece-se um relacionamento íntimo.

Caso apresentem tolerância reduzida à punição, ou em cuja história, as demonstrações afetivas foram punidas de forma frequente e severa, muitos podem sentir ambivalência, desconforto e até aversão, ao comportar-se de forma íntima (Cordova \& Scott, 2001). Geralmente estas pessoas se esquivam destes sentimentos aversivos e dificilmente ingressam em relacionamentos íntimos e afetivos por não saberem como se comportar ou por não suportarem a possibilidade de que seus comportamentos sejam punidos, o que é relatado frequentemente por indivíduos que têm psoríase.

Em resumo, as dificuldades apresentadas pelas pessoas com psoríase nas interações sociais podem ser consideradas como produtos de situações punitivas como críticas, ofensas e brincadeiras das pessoas à configuração de sua pele. Indivíduos com doença de pele, ao recusarem-se ingressar ou consolidar relacionamentos íntimos, por exemplo, estariam tentando evitar possíveis rejeições, críticas ou avaliações negativas de seus parceiros. Porém, mesmo evitando as possíveis punições, muitos continuam experimentando intenso sofrimento ao se sentirem inadequados diante das situações em que seus comportamentos eventualmente foram punidos (Mingnorance \& cols., 2001; Mingnorance \& cols., 2002; Rapp, Feldman, Exum, Fleischer \& Reboussin, 1999; Vardy \& cols., 2002).

Ressalta-se, portanto, a necessidade de análises molares dos comportamentos apresentados pelas pessoas com psoríase, visto que a fuga e a esquiva das interações sociais estão inseridas em uma complexa rede comportamental produzindo diversas consequências e relacionadas a diversos eventos. Desse modo, intervenções comportamentais pontuais e focadas em recortes muito limitados ou nas consequências imediatas nem sempre surtem os resultados mais eficazes sobre o comportamento (Zamignani \& Banaco, 2004). 


\section{CONSIDERAÇÕES FINAIS}

A literatura sobre doença de pele é vasta em citar os problemas de isolamento social e de esquiva de interações sociais, na medida em que o comportamento da comunidade verbal torna-se aversivo para o indivíduo com psoríase (críticas e ofensas à sua aparência física). Neste sentido, conhecer o repertório de comportamentos pró-sociais desses indivíduos pode ser um caminho inicial para estudar formas alternativas de prevenir o isolamento social das pessoas com esta doença. Por isso, os estudos em análise do comportamento que se dediquem a identificar quais as dificuldades apresentadas por estas pessoas e como elas as enfrentam são necessários não só para auxiliá-las na aprendizagem de comportamentos que aumentem a probabilidade de reforço positivo a curto e longo prazo e, consequentemente, prevenir problemas pessoais, profissionais e afetivos, mas também para desenvolver propostas efetivas de pesquisa e de prestação de serviços a esta população.

Muitos autores citam que há diferenças individuais no enfrentamento da psoríase (Abadie \& cols., 1994; Kimball \& cols., 2005; Leary \& cols., 1998), mesmo quando os aspectos clínicos da doença são semelhantes. Essas diferenças consistem em formas singulares de enfrentá-la. Algumas pessoas apresentam muitas dificuldades interpessoais, limitações e problemas emocionais em função de sua doença, outras, porém, não apresentam tanto comprometimento em sua vida.

A subjetividade, para a análise do comportamento é socialmente construída por meio das práticas das comunidades verbais nas quais o indivíduo interage. Entende-se por subjetividade a idiossincrasia dos indivíduos, o que os torna únicos, singulares, humanos. Desse modo, são nos contextos sociais que os indivíduos se tornam humanos, aprendem a responder discriminativamente a seus sentimentos, pensamentos, enfim, aos seus eventos públicos e privados, nomeando-os. É, portanto, nas relações sociais que aprendem como se comportar no ambiente físico e no social, aprendem sobre si, seus pensamentos e seus modos de ação. Quanto mais variados forem os contextos de interação dos indivíduos (família, grupos de amigos, colegas de trabalho, namorados), há mais possibilidade de desenvolverem comportamentos mais eficazes, sobretudo nas interações sociais e de exposição a reforçadores.

Vale salientar que contextos sociais reforçadores não são aqueles que eliminam as dificuldades nas vidas dos indivíduos, mas sim aqueles que os ensinam a como enfrentá-las, por meio de interações nas quais são incentivados a exporem-se a atividades, valorizando as suas tentativas e auxiliando-os em suas dificuldades. Contextos reforçadores são aqueles que discutem sobre a doença, que reconhecem as limitações dos indivíduos com doença de pele, mas que o valorizam a despeito de sua aparência física e reconhecem as suas potencialidades em outras atividades compatíveis com suas limitações. Por esta razão, desenvolver comportamentos pró-sociais é um passo fundamental para combater o isolamento social e contribuir para o engajamento das pessoas com psoríase em variados contextos sociais.

Ao investigar a relação entre psoríase e problemas de interação social, a preocupação do analista do comportamento deverá, então, ser a de identificar relações entre o comportamento considerado como problema e variáveis do ambiente físico e social responsáveis pela sua manutenção e tentar produzir mudanças, melhorando a interação do indivíduo que tem psoríase com sua vida e com as demais pessoas nos contextos dos quais ele faz parte.

\section{REFERÊNCIAS}

Abadie, M. S. A, Kent, G. G., \& Gawkrodger, D. J. (1994). The relationship between stress and the onset and exacerbation of psoriasis and other skin conditions. British Journal of Dermatology, 130, 199-203.

Abib, J. D. (2004). O que é comportamentalismo? Em M. Z .S. Brandão e cols. (Orgs.), Sobre comportamento e cognição: Vol. 13 - Contingências sócio-verbais e o comportamento do terapeuta (pp.52-61). Santo André: ARBytes.

Amaral, V. L. A. R., Bravo, M. C. M., \& Messias, T. C. (1996). Desenvolvimento de habilidades sociais em adolescentes portadores de deformidades faciais. Estudos de Psicologia, 13(3), 31-47.

Baum, W. M. (1999). Compreender o behaviorismo: Ciência, comportamento e cultura (M. T. A. Silva, M. A. Matos, G. Y. Tomanari, \& E. Z. Tourinho, Trad.). Porto Alegre: Artmed.

Benrud-Larson, L., Heinberg, L. J., Boling, C., Reed, J., White, B.,Wigley, F., e cols. (2003). Body image dissatisfaction among women with escleroderma: Extent and relationship to psychosocial function. Health Psychology, 22(2), 130-139.

Bilks, M. R., \& Mark, K. A. (1998). Mind-body medicine: Pratical applications in dermatology. Archives in Dermatology, 134, 1437-1441.

Capoore, H. S., Payne, C. M. E. R., \& Goldin, D. (1998). Does psychological intervention help chronic skin conditions? The Fellowship of Postgraduate Medicine, 74, 662-664.

Castro, M. G., Andrade, T. M. R., \& Muller, M. C. (2006). Conceito mente e corpo através da história. Psicologia em Estudo, 11(1), 39-43.

Catania, A. C. (1999). Aprendizagem: Comportamento, linguagem e cognição ( $4^{\mathrm{a}}$ ed.) (D.G. de Souza e cols., Trad.). Porto Alegre: Artes Médicas. 
Chiesa, M. (1994). Radical behaviorism: The philosophy and the science. Boston: Authors Cooperative.

Cordova, J. V. L., \& Scott, R. (2001). Intimacy, a behavior interpretation. The Behavior Analyst, 24(1), 75-86.

Finlay, A. (2001). Psoriasis from the patient's point of view. Archives in Dermatology, 137, 352-353.

Fortune, D. G., Richards, H. L., Main, C. J., \& Griffiths, C. E. M. (1998). What patientes with psoriasis believe about their condition. Journal of the American Academy of Dermatology, 39(2), 196-201.

Fried, R. G., Gupta, M. A., \& Gupta, A. K. (2005). Depression and skin disease. Dermatologic Clinics, 23, 657-664.

Ginsburg, I. H., \& Link, B. G. (1989). Feelings of stigmatization in patients with psoriasis. Journal of the American Academy of Dermatology, 20, 53-63.

Gupta, M. A., \& Gupta, A. K. (2004). Stressful major life events are associated with a higher frequency of cutaneous sensory symptoms: An empirical study of non-clinical subjects. Journal European Dermatology and Venereology, 18(5), 565-570.

Hayes, S. C. A (1987). Contextual approach to therapeutic change. Em N. Jacobson (Org.), Psychotherapists in clinical pratice: Cognitive and behavioral perspectives (pp. 327-387). New York: Guilford.

Hoffman, F. S., Zogbi, E., Fleck, P., \& Muller, M. C. (2005). A integração mente e corpo em psicodermatologia. Psicologia: Teoria e Prática, 7(1), 51-60.

Hünziker, M. H. L. (1997). O desamparo aprendido e a análise funcional da depressão. Em D. R. Zamignani (Org.), Sobre comportamento e cognição: Vol. 3 - Aplicação da análise do comportamento e da terapia cognitivo-comportamental no hospital geral e nos transtornos psiquiátricos (pp. 141-149). Santo André: ARBytes.

Kimball, A. B., Jacobson, C., Weiss, S., Vreeland, M. G., \& Wu, Y. (2005) The psychosocial burden of psoriasis. American Journal Clinical Dermatology, 6(6), 384-392.

Knobe, M. M. (2004). A palavra da pele. Revista FAMECOS: Midia, Cultura e Tecnologia, 1(25), 127-137.

Koblenzer, C. (1995). Psychoterapy for intractable inflammatory dermatoses. Journal of the American Academy of Dermatology, 32(4), 609-612.

Koblenzer, C. (1996). Noncutaneous manifestations of skin diseases. Psychological Aspects of Skin Disease, 41, pp. 475486.

Koo, J. Y. M., Do, J. H., \& Lee, C. S. (2000). Psychodermatology. Sinopses, 5(43), 848-853.

Krueger, G., Koo, J., Lebwohl, M., Menter, A, Stern, R. S., \& Rolstad, T. (2001). The impact of psoriasis on quality of life. Archives in Dermatology, 137, 280-284.

Leary, M. R., Rapp, S. R., Herbst, K. C., Exum, M. L., \& Feldman, S. R. (1998). Interpersonal concerns and psychological difficulties of psoriasis patients: Effects of disease severity and fear of negative evaluation. Health Psychology, 17(6), 530-536.

Marot, R. S. V. (1993). Distúrbios de ansiedade e depressão na dermatologia. Anais Brasileiros de Dermatologia, 68, 251-253.

Michael, J. (1975). Positive and negative reinforcement, a distinction that is no longer necessary; Or a better way to talk about bad things. Em E. Ramp \& G. Semb (Orgs.), Behavior analysis: Research and application (pp. 33-44). Englewood Cliffs, NJ: Prentice-Hall.

Millenson, J. R. (1975). Princípios de análise do comportamento (A. A. Souza e D. Rezende, Trad.). Brasília: Coordenada.

Mingnorance, R. C, Loureiro, S. R., Okino, L., \& Foss, N. T. (2001). Pacientes com psoríase: Adaptação social e características de personalidade. Medicina, 34, 315-324.

Mingnorance, R. C., Loureiro, S. R., \& Okino, L. (2002). Pacientes com psoríase: Qualidade de vida e adaptação psicossocial. Anais Brasileiros de Dermatologia, 77(2), 147159.

Montagu, A. (1986). Tocar: O significado humano da pele (M. S. M. Netto, Trad.) São Paulo: Summus.

Ongenae, K., Beelaert, L., Geel, N. V., \& Naeyaert, J. M. (2006). Psychosocial effects of vitiligo. Journal of European Academy of Dermatology and Venereology, 20, 1-8.

Patrus, O. A. (1991). Fatores psicogênicos em psoríase: Argumentos favoráveis. Anais Brasileiros de Dermatologia, 66, 31-33.

Perone, M. (2003). Negative effects of positive reinforcement. The Behavior Analyst, 1(1), 1-14.

Picardi, A., \& Abeni, D. (2001a). Can cognitive-behavioral therapy help patients with vitiligo? Archives in Dermatology, 137(6), 788-791.

Picardi, A., \& Abeni, D. (2001b). Stressfull life events and skin diseases: Disentangling evidence from myth. Psichotherapy and Psychosomatics, 70, 118-136.

Picardi, A., Mazzotti E., Gaetano P., Cattaruzza, M. S., Baliva, G., Melchi, C. F., e cols. (2005). Stress, social support, emotional regulation, and exacerbation of diffuse plaque psoriasis. American Psychiatric Publishing, 46(6), 9.

Porter, J. R., Beuf, A. H., Lerner, A. B., \& Nordlund, J. J. (1990). The effect of vitiligo on sexual relationships. Journal American Academy of Dermatology, 22, 221-222.

Rapp, S. R., Feldman S. R., Exum, M. L., Fleischer, A. B. J., \& Reboussin, D. M. (1999). Psoriases causes as much disability as other major medical diseases. American Academy of Dermatology, 41, 401-407.

Rapp, S. R., Cottrell, C. A., \& Leary, M. R. (2001). Social coping strategies associated whit quality of life decrements among psoriaris patients. British Journal of Dermatology, 145, 610616.

Renzi, C., Abeni, D., Picardi, A., Agostini, E., Melchi, C. F., Pasquini, P., e cols. (2001). Factors associated with patient satisfaction with care among dermatological outpatients. British Journal of Dermatology, 145, 617-623.

Rosmaninho, I., Fernandes, L., Miranda, M., de Olivera, J. F., Lopez, J. M., \& Vaz, M. (2003). Dermatose neuropsicogênica. Revista Portuguesa de Psicossomática, 5(1), 101-104.

Savin, J. A., \& Cotterill, J. A. (1992). Psychocutaneous disorders. Em R. H. Champion e cols. (Orgs.), Textbook of dermatology (pp. 2479-2496). Oxford: Blackwell.

Seng, T. K., \& Nee, T. S. (1997). Group therapy: A useful and supportive treatment for psoriasis patients. Blackwell Science, $36,110-112$. 
Sidman, M. (1995). Coerção e suas implicações (M. A. P. A. Andery \& T. M. A. P. Sério, Trad.). Campinas: Psy II.

Silva, K. S., \& Silva, E. A. T. (2007). Psoríase e sua relação com aspectos psicológicos, stress e eventos da vida. Estudos de Psicologia, 24( 2),1-13.

Skinner, B. F. (1965). Science and human behavior. New York: Macmillan

Skinner, B. F. (1974). About behaviorism. New York: Alfred A. Knop.

Taborda, M. L. V. V., Weber, M. B., \& Freitas, E. S. (2005). Avaliação da prevalência de sofrimento psíquico em pacientes com dermatoses do espectro dos transtornos psicocutâneos. Anais Brasileiros de Dermatologia, 80(4), 351-354.

Takahashi, M. D. F. (1991) Influência dos fatores psicogênicos na psoríase. Anais Brasileiros de Dermatologia, 66, 34.

Taylor, S. E. (1997). Healthy psychology: What is an unhealthy environment and how does it get under the skin? Psychological Review, 48, 411-447.

Vardy, D., Besser, A., Amir, M., Gesthater, B., Biton, A., \& Buskila, D. (2002). Experiences of stigamatization play a role in mediating the impact of disease severity on quality of life in psoriasis patients. British Association of Dermatology, 147(40), 736-742.

Whal, A., Loge, J. H., Wiklund, I., Hanestad, B. R., \& Trondheim, N. (2000). The burden of psoriasis: A study concerning helthrelated quality of live among norwegian adult patients with psoriasis compared with general population norms. Journal American Academy of Dermatology, 43, 803-808.

Zachariae, R., Oster, H; Bjerring, P., \& Kragballe, K. (1996). Effects of psychologic intervention on psoríase: A preliminary report. Journal of the American Academy of Dermatology, 34(6), 1008-1015.

Zamignani, D. R., \& Banaco, R. A. (2004). An analitycal behavioral panorama on the anxiety disorders. Em T. C. Grassi (Org.), Contemporany challenges in the behavioral approach: A Brazilian overview (pp. 9-26). Santo André: Esetec.

Recebido: $15 / 02 / 2008$

Última revisão: 26/08/2008 Aceite final: 07/10/2008

\section{Notas:}

1 Herança ambiental está sendo utilizada em contraposição à origem genética da doença dermatológica.

2 A literatura médico-psiquiátrica refere-se a fatores de natureza psicológica de uma forma bastante abrangente, incluindo neste conceito, os transtornos psiquiátricos, as dificuldades interpessoais, as emoções, os fatores de estresse, o enfrentamento de situações aversivas, coping e fatores psicossocias. No decorrer do trabalho, os fatores psicológicos serão melhor detalhados. Considera-se que, para a análise do comportamento, fatores psicológicos equivalem-se a fatores comportamentais, na medida em que comportamento inclui todas as atividades de um organismo vivo, ainda que estas atividades estejam acessíveis apenas ao próprio indivíduo (pensamento, sentimentos) (Chiesa, 1994; Skinner, 1974).

\section{Sobre os autores:}

Carolina Martinez Sampaio Mota: Especialista em Psicoterapia na Análise do Comportamento pela Universidade Estadual de Londrina. Mestranda em Análise do Comportamento - UEL. Professora Colaboradora do Departamento de Psicologia Geral e Análise do Comportamento da Universidade Estadual de Londrina.

Márcia Cristina Caserta Gon: Doutora em Psicobiologia pela Universidade de São Paulo. Professora Adjunta do Departamento de Psicologia Geral e Análise do Comportamento da Universidade Estadual de Londrina.

Airton dos Santos Gon: Mestre em Medicina pela Universidade Estadual de Londrina. Professor Assistente do Departamento de Clínica Médica da Universidade Estadual de Londrina / Clínica de Dermatologia.

Endereço para correspondência: airton@sercomtel.com.br. 